\title{
Predação de Ramphocelus carbo (Pallas, 1764) por Oxybelis fulgidus (Daudin, 1803) no Sudoeste da Amazônia brasileira
}

Relatamos neste trabalho uma observação de predação de Ramphocelus carbo (Pallas, 1764) por Oxybelis fulgidus (Daudin, 1803) na área rural da cidade de Espigão D'Oeste, estado de Rondônia, Brasil, no sudoeste da Amazônia brasileira. O local é um sítio particular, em uma plantação de Café (Coffea canephora). A O. fulgidus predou o Ramphocelus carbo numa altura de aproximadamente $120 \mathrm{~cm}$ do solo. Este relato de comportamento é de interesse pois demonstra o hábito de serpentes arborícolas de caçar próximo a fontes de alimento de suas presas (Ramphocelus carbo), assim aumentando a probabilidade de encontrar presas como aves.

Palavras-chave: Rondônia; Ave; Cobra; Presa; Floresta amazônica.

\section{Predation of Ramphocelus carbo (Pallas, 1764) by Oxybelis fulgidus (Daudin, 1803) in Southwestern Brazilian Amazon}

We report an observation of predation on Ramphocelus carbo (Pallas, 1764) by Oxybelis fulgidus (Daudin, 1803) in the rural area of the city of Espigão D'Oeste, Rondônia state, Brazil, in the southwestern Brazilian Amazon. The site is a private farm, in a coffee (Coffea canephora) plantation. O. fulgidus preys on Ramphocelus carbo at a height of about $120 \mathrm{~cm}$ from the ground. This behavior report is of interest because it demonstrates the habit of arboreal snakes to hunt near food sources of their prey (Ramphocelus carbo), thus increasing the probability of finding prey like birds.

Keywords: Rondônia; Bird; Snake; Prey; Amazon forest.

Topic: Notas Científicas

Reviewed anonymously in the process of blind peer.
Received: 06/03/2021

Approved: $27 / 05 / 2021$

Raul Afonso Pommer Barbosa ib

Fundação Getúlio Vargas, Brasil

http://lattes.cnpq.br/9136549262197723

http://orcid.org/0000-0002-1914-5184

raulpommer@hotmail.com

Geovanna Santos da Silva (it)

Centro Universitário São Lucas, Brasil

http://lattes.cnpq.br/3780108256426220

http://orcid.org/0000-0001-5881-622X

geovannas487@gmail.com

Marcela Alvares Oliveira (iD

Universidade Federal de Rondônia, Brasil

http://lattes.cnpq.br/9346965102777187

http://orcid.org/0000-0002-4129-993X

marcela.mugrabe@gmail.com

Referencing this:

BARBOSA, R. A. P.; SILVA, G. S.; OLIVEIRA, M. A.. Predação de Ramphocelus carbo (Pallas, 1764) por Oxybelis fulgidus (Daudin, 1803) no Sudoeste da Amazônia brasileira. Nature and Conservation, v.14, n.2, p.219-223, 2021. DOI: http://doi.org/10.6008/CBPC23182881.2021.002.0020 


\section{INTRODUÇÃO}

O registro de eventos de predação em ambientes naturais é raro, principalmente o de animais como serpentes que possuem o hábito furtivo (OLIVEIRA, 2007). Eventos de predação são um dos fatores que influenciam o sucesso reprodutivo das espécies de aves e a estrutura das comunidades (RICKLEFS, 1969; WIENS, 1994).

A green vine snake, Oxybelis fulgidus (Daudin, 1803), é uma serpente que pertence à família Colubridae, arborícola e diurna (HENDERSON, 1982) que se distribui do sul do México até a região central do Brasil (VILLA et al., 1995), sendo uma espécie comum na Amazônia Brasileira (CAPURUCHO et al., 2012). Pode ser encontrada em uma grande variedade de ambientes, que incluem fragmentos florestais urbanos, vegetação ripária, bordas florestais, floresta de pinheiro, plantações (LOVICH et al., 2010; FRAGA et al., 2012; RODRÍGUEZ-PÉREZ et al., 2019; ALMEIDA et al., 2020). Embora ocorra essa amplitude tipos de ambientes utilizados, incluindo espaços antrópicos, os acidentes com humanos são raramente reportados (SILVA et al., 2019). Contudo, devido ao seu padrão de coloração verde e a proximidade com áreas urbanas, pode ser eventualmente confundida com a Bothrops bilineatus e ser morta por essa razão (FERMIANO et al., 2018).

Os machos alcançam a maturidade sexual com um comprimento de $710 \mathrm{~mm}$, enquanto as fêmeas alcançam a maturidade com 900 mm (SCARTOZZONI et al., 2009). São ovíparas, tendo registro de seis e sete ovos por evento de ovoposição ( 44,9 X 18,6 mm e 11,6 g de peso) com um período de incubação de 98,6 \pm 1,1 dias e os filhotes eclodindo com 356,7 mm de comprimento e pesando 6,8 g, em média (GROVES, 1995; FIGUEROA et al., 2013).

Os itens da composição da dieta da $O$. fulgidus têm sido definidos principalmente através de registros de predação. A dieta é composta com aves, lagartos e pequenos mamíferos (NORRIS et al., 1998; SOSABARTUANO et al., 2015; CARBAJAL-MÁRQUEZ et al., 2019; RODRÍGUEZ-PÉREZ et al., 2019; ALMEIDA et al., 2020; BROWN, 2020). Essa preferência pode estar relacionada com a toxicidade do seu veneno, já que ele apresenta elevada toxicidade letal para lagartos e aves, mas não é tóxica para mamíferos (HEYBORNE et al., 2013). Em relação às aves, existe o registro de predação de espécies pertencentes a 18 famílias (BROWN, 2020), demonstrando ser um comportamento de caça altamente oportunista e um excelente caçador de emboscada, sendo sua dependente de suas capacidades visuais e olfativas (GROVES, 1995).

A Pipira-vermelha é do gênero Ramphocelus que engloba dez espécies, porém, apenas três espécies ocorrem no Brasil, sendo elas R. carbo, R. nigrogularis e R. bresilius (HILTY, 2018; PIACENTINI, 2015). Ramphocelus carbo (Pallas, 1764), a pipira-vermelha, é um pássaro amplamente distribuído na América do Sul que vive em arbustos nas bordas da floresta, lareiras cobertas de vegetação, plantações ao redor de moradias e vegetação densa de arbustos ao longo das margens dos rios em áreas úmidas e, especialmente, em áreas antropizadas (HILTY, 2018; SOUZA et al., 2020).

Formam pequenos bandos, predominando apenas um sexo, macho ou fêmea, e em horários quentes permanece nas árvores, à meia altura e quietos (CARVALHO, 1957). A alimentação tanto dos adultos como dos filhotes, consiste em semente de paxiúba (Socratea exorrhiza Mart.), frutos de antúrio epifítico 
(Anthurium sp.), abiu (Pouteria caimito Ruiz \& Pavon), sapoti (Achras .sapota L.), bem como banana e goiaba (CARVALHO, 1957).

\section{RELATO}

Realizamos observações de campo no município de Espigão D'Oeste, estado de Rondônia, Brasil, no sudoeste da Amazônia brasileira. O evento de predação do Ramphocelus carbo (Pallas, 1764) por Oxybelis fulgidus (Daudin, 1803) foi observado, às 15:00 PM do dia 2 de fevereiro de 2021. O local é um sítio particular, em uma plantação de Café (Coffea canephora). A O. fulgidus predou o Ramphocelus carbo numa altura de aproximadamente $120 \mathrm{~cm}$ do solo, sobre um Coffea canephora (Figura 1). Este relato de comportamento é de interesse pois demonstra o hábito de serpentes arborícolas de caçar próximo a fontes de alimento de suas presas (Ramphocelus carbo), assim aumentando a probabilidade de encontrar presas como aves. 7

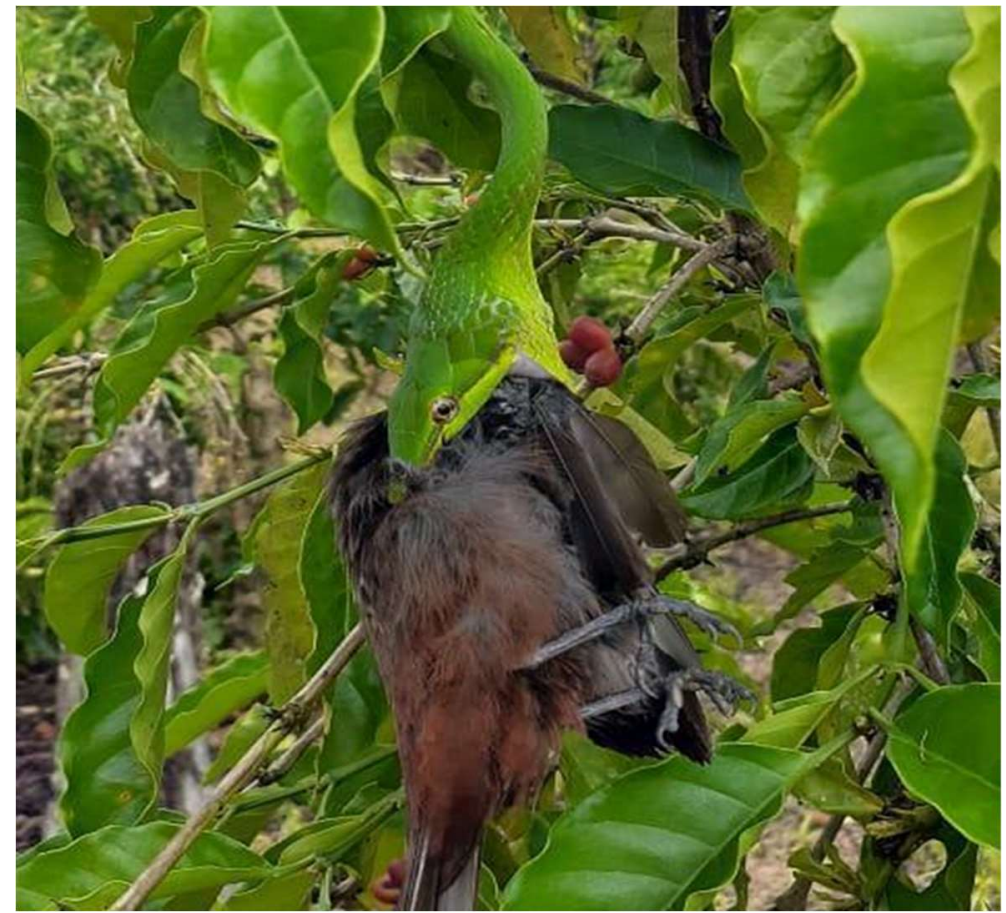

Figura 1: Evidência fotográfica da Predação da Ramphocelus carbo (Pallas, 1764) por Oxybelis fulgidus (Daudin, 1803).

\section{DISCUSSÃO}

Anteriormente a esse registo, havia dois registros de predação de Ramphocelus carbo por Oxybelis fulgidus localizados na porção leste (HERÁNDEZ-RUZ et al., 2019 no Brasil), e noroeste (MAY, 2019 na Colômbia) da sua distribuição na América do Sul, todos em ambientes modificados pela ação antrópica. $O$ presente relato insere um novo registro de predação na porção sul da distribuição da espécie, em condições similares aos dois primeiros (Figura 2). 


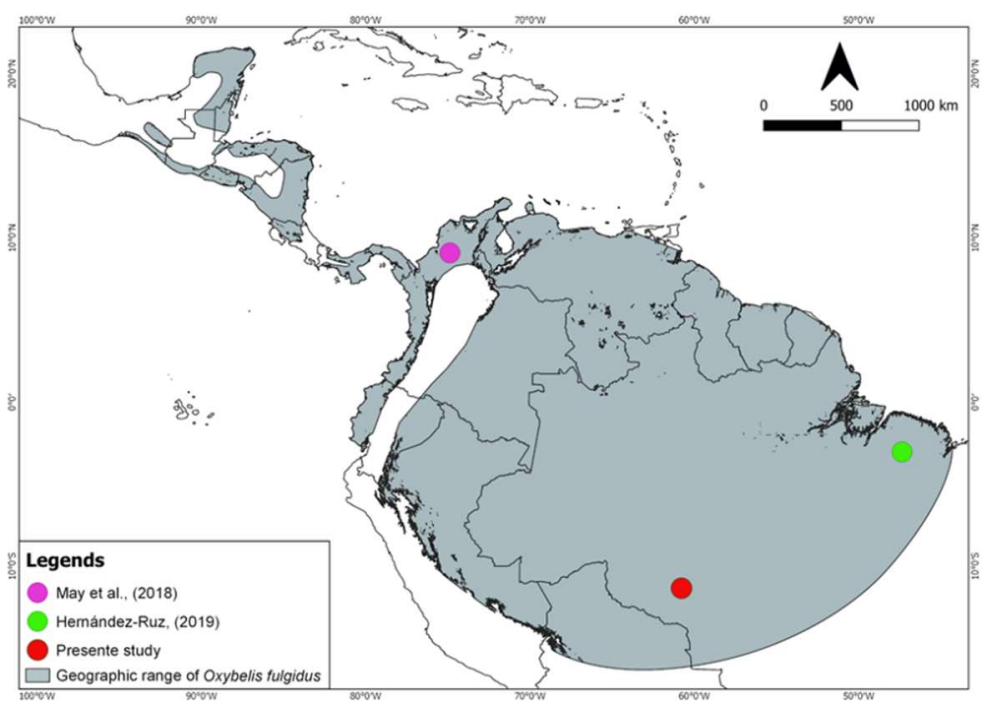

Figura 2: Registros de predação de Ramphocelus carbo (Pallas, 1764) por Oxybelis fulgidus (Daudin, 1803) ao longo de sua distribuição geográfica.

\section{CONSIDERAÇÕES FINAIS}

Ressaltamos a importância de estudos voltados para a compreensão dos hábitos alimentares de Oxybelis fulgidus, a fim de compreender melhor os padrões apresentados pela espécie e como estes contribuem para o equilíbrio de ecossistemas. Registros deste tipo ampliam o conhecimento sobre os hábitos alimentares das $O$. fulgidus em vida livre e são importantes para aumentar o conhecimento sobre os predadores da espécie Ramphocelus carbo.

AGRADECIMENTOS: Nós agradecemos ao Marcos Knidel e a Alidia Zilske pelo registro fotográfico utilizado neste trabalho. Agradecemos a Ana Paula Vitória Costa Rodrigues pela identificação da serpente. Agradecemos ao "Projeto Que Mico é Esse?" pelo apoio e suporte financeiro. Agradecemos ao Clube de Astronomia e Ciências de Rondônia pelo apoio e suporte financeiro. Agradecemos ao Centro de Cultura e Formação Kanindé pelo apoio logístico. Agradecemos a Coordenação de Aperfeiçoamento de Pessoal de Nível Superior - CAPES pelo apoio financeiro.

\section{REFERÊNCIAS}

ALMEIDA, L. S.; SILVA, A. W. O.; TREVINE, V.. Two new records of predation by Oxybelis fulgidus (Squamata: Colubridae) in the northern region of Brazil. Herpetology Notes, v.13, p.283-289, 2020.

BROWN, T. W..Predation of Bay-breasted Warbler Setophaga castanea (Parulidae) by Green Vinesnake Oxybelis fulgidus (Colubridae) on Utila Island, Honduras. Captive \& Field Herpetology, v.4, n.1, p.41-44, 2020.

CAPURUCHO, J. M. G.; COSTA, H. C.. Oxybelis fulgidus (Green Vinesnake). Diet. Herpetological Review, v.43, n.3, p.495, 2012.

CARBAJAL-MÁRQUEZ, R. A.; GARCÍA-BALDERAS C. M.; RAMÍREZ-VALVERDE, T.; CEDEÑO-VÁZQUEZ, J. R.; BLANCOCAMPOS, V. G.. New prey items in the diet of snakes from the Yucatán Peninsula, Mexico. Cuadernos de Herpetología, v.33, n.2, p.71-74, 2019

CARVALHO, C. T.. Notas sobre a biologia do Ramphocelus carbo (Passeres, Thraupidae). Museu Goeldi, 1957.

FERMIANO, E. C.; ROBERTO, W. S.; SOUZA, J.; SILVA, P. J. G.; SURUI, F. G.; SILVA, A. P.. Serpentes registradas ocasionalmente no município de Alvorada do Oeste-RO, entre 2016 e 2017. Revista Saber Científico, v.7, n.1, p.91$98,2018$.

FIGUEROA, A.; LEWIS T. R.; MOONEY, P.. Notes on reproduction in Oxybelis fulgidus from Costa Rica. Herpetology Notes, v.6, p.29-31, 2013.

FRAGA, R.; CARVALHO, V. T.; VOGT, R. C.. Oxybelis fulgidus (Green Vinesnake). Diet. Herpetological Review, v.43, n.3, p.495-496, 2012. 
GROVES, J. D. Reproduction and feeding behavior of Oxybelis wilsoni, a new species of vine snake (Serpentes: Colubridae). Revista de Biología Tropical, v.43, n.1-3, p.307-309, 1995.

HENDERSON, R. W.. Trophic relationships and foraging strategies of some New World tree snakes (Leptophis, Oxybelis, Uromacer). Amphibia-Reptilia, v.3, n.1, p.71-80, 1982. DOI: https://doi.org/10.1163/156853882X00185

HEYBORNE, W. H.; MACKESSY, S. P.. Identification and characterization of a taxon-specific three-finger toxin from the venom of the Green Vinesnake (Oxybelis fulgidus; family Colubridae). Biochimie, v.95, n.10, p.1923-1932, 2013. DOI: https://doi.org/10.1016/j.biochi.2013.06.025

HILTY, S.. Tanager de bico de prata (Ramphocelus carbo). In: DEL HOYO, J.; ELLIOTT, A.; SARGATAL, J.; CHRISTIE, D. A.; JUANA, E.. Manual dos pássaros do mundo vivo. Barcelona: Lynx Edicions, 2018.

LOVICH, R.; AKRE, T.; RYAN, M.; NUÑEZ, S.; CRUZ, G.; BORJAS, G.; SCOTT, N. J.; FLORES, S.; DEL CID, W.; FLORES, A.; RODRIGUEZ, C.; LUQUE-MONTES, I. R.; FORD, R.. New Herpetofaunal records from southern Honduras. Herpetological Review, v.41, n.1, p.112-115, 2010.

NORRIS, J. L.; BURTT JUNIOR, E. H.. Oxybelis fulgidus (Green Vine Snake or Bejucillo). Feeding. Herpetological Review, v.29, n.4, p.243, 1998.

OLIVEIRA, C. H. P.. Predação de Ninhegos de Formicivora Littoralis (Aves: Thamnophilidae) por Pseustes Sulphureus (Reptilia: Colubridae) na Ilha de Cabo Frio, Arraial do Cabo RJ. In: CONGRESSO DE ECOLOGIA DO BRASIL, 8. Anais. Caxambu, 2007.

PIACENTINI, V. Q.; ALEIXO, A.; AGNE, C. E.; MAURICIO, G. N.; PACHECO, J. F.; BRAVO, G. A.. Lista de verificação comentada das aves do Brasil pelo Comitê Brasileiro de Registros Ornitológicos. Rev. Bras. Ornitol, v.23, n2 p.91-298, 2015.
RICKLEFS, R. E.. An analysis of nesting mortality in birds. Smithsonian Contributions to Zoology, n.9, p.1-48, 1969.

RODRÍGUEZ-PÉREZ, C.; MATA-SILVA, V.. Oxybelis fulgidus (Green Vinesnake). Diet and habitat. Herpetological Review, v.50, n.1, p.166, 2019.

SCARTOZZONI, R. R.; SALOMÃO, M. G.; SANTOS, S. M. A. Natural history of the vine snake Oxybelis fulgidus (Serpentes, Colubridae) from Brazil. South American Journal of Herpetology, v.4, n.1, p.81-89, 2009. DOI: https://doi.org/10.2994/057.004.0111

SILVA, K. V.; SAID, R. C.; ASSY, J. G. P. L.; DUARTE, M. R.; TORREZ, P. P. Q.; FRANÇA, F. O. S.. A case of envenomation caused by Oxybelis fulgidus (Serpentes, Colubridae) in Brazilian Amazon. Revista da Sociedade Brasileira de Medicina Tropical, v.52, e20180423, 2019. DOI: https://doi.org/10.1590/0037-8682-0426-2018

SOSA-BARTUANO, A.; RODRÍGUEZ-BEITÍA, B.. Oxybelis fulgidus: Diet. Mesoamerican Herpetology, v.2, n.4, p.527528, 2015.

SOUZA, L. S.; GUILHERME, E.; GUL, S.; ANDRADE, A. M. F.; SANTOS, F. G. A.. Primeiro relato de Davaneidae e Strongylida parasitando Ramphocelus carbo (Aves: Passeriformes: Thraupidae) no sudoeste da Amazônia brasileira. Rev. Bras. Parasitol. Veterinario., Jaboticabal, v.29, n.1, e016719, 2020. DOI: https://doi.org/10.1590/s1984-29612019105

VILLA, J. D.; MCCRANIE, J. R.. Oxybelis wilsoni, a new species of vine snake from Isla de Roatán, Honduras (Serpentes: Colubridae). Revista de Biologia Tropical, v.43, n.1-3, p.297305, 1995.

WIENS, J. A.. The ecology of bird communities. Cambridge: Cambridge University Press, 1994.

A CBPC - Companhia Brasileira de Produção Científica (CNPJ: 11.221.422/0001-03) detém os direitos materiais desta publicação. Os direitos referem-se à publicação do trabalho em qualquer parte do mundo, incluindo os direitos às renovações, expansões e disseminações da contribuição, bem como outros direitos subsidiários. Todos os trabalhos publicados eletronicamente poderão posteriormente ser publicados em coletâneas impressas sob coordenação da Sustenere Publishing, da Companhia Brasileira de Produção Científica e seus parceiros autorizados. Os (as) autores (as) preservam os direitos autorais, mas não têm permissão para a publicação da contribuição em outro meio, impresso ou digital, em português ou em tradução. 